\title{
FORMACIÓN DIDÁCTICA L2 (KAQCHIKEL) EN LA ENBI NIW NA’OJ
}

\section{DIDACTIC TRAINING L2 (KAQCHIKEL) AT ENBI NIW NA'OJ}

\section{Referencia del artículo}

Chalí, L. (2020). COVID-19: Formación didáctica L2 (Kaqchikel) en la ENBI Niw Na'oj. Revista Científica del SEP. 3(1), 39-44. DOI: https://doi.org/10.36958/sep.v3i01.31

\author{
Lilian Esterlina Chalí Sarech \\ liliesterlyn@gmail.com \\ Profesora de Enseñanza Media \\ Escuela de Formación de Profesores de Enseñanza Media EFPEM \\ Universidad de San Carlos de Guatemala \\ Fecha de recibido: $15 / 06 / 2020$ \\ Fecha de aceptado: 04/11/2020
}

\section{RESUMEN}

Este artículo presenta los resultados de la investigación titulada" Formación didáctica L2 (kaqchikel) en la ENBI Nim Na'oj", realizada en enero y febrero de 2020.

La investigación tuvo como objetivo establecer las estrategias de enseñanza-aprendizaje que utiliza el docente en la formación didáctica que reciben los estudiantes de Magisterio Infantil Bilingüe Intercultural, para el abordaje la Segunda Lengua - L2 (kaqchikel). Los resultados muestran que tanto los documentos así- también el docente no cuenta con una metodologí-a definida y técnicas adecuadas para el desarrollo de los contenidos didácticos propuestos en las sub-áreas didáctica L2.

Ante esta situación, existe la necesidad de actualización docente en el uso y domino de las cuatro competencias lingüí-sticas del Kaqchikel - L2 para desarrollo del curso de Didáctica, que facilite la interacción entre los estudiantes y docentes. El principal desafí-o de la Escuela Normal de Educación Bilingüe Intercultural Nim Na'oj será promover una educación de calidad que responda a las caracterí-sticas culturales de las comunidades indí-genas para asegurar el derecho de ser educados en su propia cultura en dos o más idiomas.

\section{PALABRAS CLAVE}

Metodología, educación, calidad, competencia y lingüística

\section{ABSTRACT}

This article presents the results of the research entitled "L2 didactic training (kaqchikel) at ENBI Nim Na'oj", carried out in January and February 2020.

The objective of the research was to establish the teachinglearning strategies used by the teacher in the didactic training received by students of Intercultural Bilingual Early Childhood Teaching, for the Second Language - L2 (kaqchikel) approach. The results show that both the documents as well as the teacher does not have a defined methodology and adequate techniques for the development of the didactic contents proposed in the L2 didactic sub-areas.

Faced with this situation, there is a need to update teachers in the use and mastery of the four linguistic competences of Kaqchikel - L2 for the development of the Didactics course, which facilitates interaction between students and teachers. The main challenge of the Nim Na'oj Intercultural Bilingual Education Normal School will be to promote quality education that responds to the cultural characteristics of indigenous communities to ensure the right to be educated in their own culture in two or more languages.

\section{KEYWORDS}

Methodology, education, quality, competence and linguistics 


\section{INTRODUCCIÓN}

El presente estudio se realizó por el bajo nivel de bilingüismo que se observó en los en los estudiantes de la ENBI Nim Na'oj, a pesar de pertenecer a un área kaqchikel y que su idioma materno es el español. Históricamente la mayoría de la población es de origen kaqchikel, pero fue perdiendo el uso y práctica de su idioma en los años 50 y 60 con el programa de castellanización y por el fenómeno de la discriminación racial. De hecho esta situación de pérdida lingüística en el Municipio de San Martín Jilotepeque tiene consecuencias en el desarrollo educativo, especialmente en el caso de la formación docente de la ENBI Nim Na'oj.

La investigación reside sobre la importancia del proceso metodológico de enseñanza-aprendizaje recibido por los estudiantes puesto que no se desarrollan de manera bilingüe por lo tanto el problema que dio origen a este estudio es el bajo nivel de bilingüismo de los futuros docentes de educación infantil.

La formación inicial docente ha sido un reto educativo por medio del cual se puede mejorar el proceso de aprendizaje de los estudiantes, de cambiar los planes de estudios o currículos que se aplican en las escuelas, de introducir la innovación didáctica y metodológica (Elbaz 1983, p.11).

Este estudio es relevante en el área de conocimiento de los estudiantes de esta carrera porque se establece en el pensum de estudio la sub-área de la didáctica de L2 lo cual busca el desarrollo de las habilidades comunicativas del aprendizaje de un idioma materno, con actividades que le sean familiares y atractivas para facilitar su aprendizaje.

Por lo que se toma como objetivo general de establecer las estrategias de enseñanza-aprendizaje que utilizan los docentes en la formación didáctica que reciben los estudiantes de Magisterio Infantil Bilingüe Intercultural para el abordaje de la L2 (kaqchikel).

Es necesaria la aplicación de la didáctica para mejoramiento del proceso de enseñanza aprendizaje que el estudiante espera recibir. Tal como lo explica Chevallard al referirse al proceso de transposición del conocimiento, citado por Mugrabi (2019), "para que la enseñanza de un elemento particular del conocimiento sea posible, éste deberá someterse a ciertas deformaciones que lo harán apto a ser enseñado".

Así mismo se toma como objetivo específico, describir los fundamentos didácticos que proponen los documentos que utilizan los docentes en el desarrollo de las capacidades para la enseñanza de la L2 (kaqchikel) en los futuros docentes y también identificar los contenidos didácticos fundamentales en la sub-área de Didáctica del segundo idioma L2 (kaqchikel), en los documentos que orientan a los estudiantes de Magisterio Infantil Bilingüe Intercultural.

Se hace una descripción y análisis de la problemática que a partir del cuestionario se obtuvo el resultado de la investigación realizada.

El problema de la formación didáctica inicial de los futuros docentes está relacionado a diversos factores y elementos tales como: La estructura y organización de la formación docente;

a) Las disciplinas de los contenidos,

b) Las actividades pedagógicas y

c) Los puntos de vistas desde distintas perspectivas (ministros/político, etc.).

La formación didáctica de los docentes en la etapa inicial está vinculada a la construcción de competencias curriculares y lingüísticas para producir aprendizajes específicos de carácter disciplinar.

Ante la problemática tanto de la estructura y organización de la formación docente hasta las actividades pedagógicas se planteó el objetivo de investigación, que es establecer las estrategias de enseñanza-aprendizaje que utilizan los docentes en la formación didáctica que reciben los estudiantes de Magisterio Infantil Bilingüe Intercultural, para el abordaje de la L2 (kaqchikel).

Se utilizaron los siguientes instrumentos: cuestionario, guía de observación y lista de cotejo para observar las actividades desarrolladas en el aula. Los sujetos fueron los estudiantes del grado de sexto magisterio infantil bilingüe intercultural y el docente que imparte la sub-área de Didáctica de L2, con una población de 27 estudiantes y un docente de la ENBI Nim Na'oj. Por la cantidad de sujetos, se tomó la población total y no se seleccionó muestra.

Los resultados de la investigación no fueron tan alentadores, hay carencia de uso de estrategias y materiales didácticos para la enseñanza del idioma kaqchikel.

Las estrategias didácticas en este nivel educativo son fundamentales para los docentes que acompañan a los estudiantes en este proceso, por ello se hace necesaria la aplicación de metodologías innovadoras que permitan aprender a aprender, aprender a ser, aprender a emprender, aprender a conocer y aprender a convivir para el desarrollo integral de la persona humana, tal como está propuesto en el Currículum Nacional Base de la carrera en mención. 
La Escuela Normal Bilingüe Intercultural tiene como responsabilidad el impulso de la calidad educativa, para satisfacer las demandas de la formación de los nuevos docentes, con un currículo y pensum de estudios con pertinencia cultural y lingüística, debido que en las manos de los mentores está el porvenir de los jóvenes y niños

La investigación se realizó en el departamento de Chimaltenango, municipio de San Martín Jilotepeque, zona 3, Barrio el Güite, específicamente en la Escuela Normal Bilingüe Intercultural Nim Na'oj, con estudiantes de la carrera de Magisterio Infantil Bilingüe Intercultural del ciclo escolar 2020.

\section{RECOLECCIÓN DE DATOS}

En la recolección de los datos no se utilizó muestra por la cantidad reducida de los sujetos investigados, se tomó la población total.

\section{TÉCNICAS E INSTRUMENTOS}

El enfoque de la investigación es mixto y con el diseño descriptivo.

En dicho estudio se utilizaron como instrumentos el cuestionario y una lista de cotejo de las actividades desarrolladas en el aula.

En la investigación se optó por la observación directa en el aula, entrevista a docente y estudiantes y análisis documental.

\section{PROCESAMIENTO Y ANÁLISIS DE INFORMACIÓN}

Con la finalidad de alcanzar los objetivos de estudio, se utilizó el método descriptivo para investigar el problema y que al seguir el procedimiento de forma correcta se obtuvo la información que constituye "como materia prima de la investigación cualitativa".

Para lo cual, se utilizó las técnicas de recopilación de datos para descubrir la situación de cómo se está desarrollando la Didáctica de L2, en la Escuela Normal Bilingüe Intercultural Nim Na'oj.

La información recabada fue el punto de partida para el proceso de sistematización de la experiencia y construcción del conocimiento, que posteriormente sirvió para comprender e interpretar la realidad que viven los miembros de la comunidad educativa, especialmente al docente y estudiantes.

En este sentido, la observadora estuvo como "participante" en el salón de clase, ya que imparte otros cursos en la carrera de
Magisterio Infantil Bilingüe Intercultural. Este hecho, coadyuvó a registrar y categorizar la información objetiva para el estudio de investigación.

Por otra parte, la aplicación de la técnica de entrevista al catedrático y a los estudiantes de dichos cursos proporcionó datos valiosos y significativos para usos de la investigación, dado el clima de confianza que estableció en la comunicación verbal provocada y guiada por la entrevistadora con un esquema de preguntas estructuradas con cierta flexibilidad.

La dinámica que se produjo entre la entrevistadora y los entrevistados se siguió cierto protocolo:

a) El acercamiento inicial para explicarle al entrevistado de que se trata la investigación y qué se espera de él o de ella.

b) Introducirle en el tema y profundizar en el mismo.

c) Se le dio respuesta a las preguntas utilizadas en la entrevista, con la opinión emitida por los entrevistados.

d) Auxiliarse de instrumentos tecnológicos para grabar la información.

e) Se sistematizó la información recabada.

f) Se analizó y seleccionó la información relevante.

Además, se utilizó la técnica documental para la recolección de información relacionado al tema de investigación, la cual coadyuvó a la construcción de marco teórico referencial y al análisis de datos de la investigación.

El material informativo permitió conocer otras investigaciones.

El procesamiento de la información se logró a través de la revisión, organización, clasificación, compilación y presentación mediante gráficas, que posteriormente sirvió para el análisis de la misma.

En el análisis de datos se empleó la triangulación de fuentes de datos obtenidos en la guía de observación, entrevista y material documental, dichas fuentes se orientaron hacia el mismo objetivo de estudio.

Las primeras fuentes de información (la guía y la entrevista) revelaron la debilidad de los docentes que imparten las clases de Didáctica de Kaqchikel (L2).

No cabe duda, los docentes carecen de conocimientos de las estrategias adecuadas de enseñanza para el aprendizaje de un segundo idioma para los estudiantes. De esta manera, los estudiantes no encuentran el sentido y significado práctico para el aprendizaje del kaqchikel. Objetivamente, no se puede hablar de bilingüismo. 
El tercero de las fuentes es material documental que nutrió las diferentes visiones, enfoques, y perspectivas teóricas de cómo se desarrolla el curso de Didáctica de L2 en la carrera Magisterio Infantil Bilingüe Intercultural en San Martín Jilotepeque.

Dicho análisis constituyó uno de los momentos más relevantes para identificar, enriquecer y profundizar los aportes esperados en la investigación. Por tanto, la triangulación permitió conocer los significados y definiciones del objeto de estudio, así como la explicación e interpretación de las causas y consecuencias que afecta el proceso educativo de los futuros docentes.

Este análisis se realizó bajo los criterios de credibilidad validez, veracidad de la información para el logro de los resultados y proyectar las conclusiones de la investigación.

\section{MARCO METODOLÓGICO}

Esta investigación se centra en la formación didáctica en L2 (kaqchikel) de los estudiantes de Magisterio Infantil Bilingüe Intercultural enfocado en el abordaje del bilingüismo.

Se orientó en el punto de vista de Mugrabi (2019), para considerar la importancia de la didáctica en la práctica del bilingüismo. Para ello se da a conocer los fines de las asignaturas que realizan procesos didácticos en los idiomas.

Así como la didáctica del segundo idioma L2, esta asignatura está diseñada para brindar a los estudiantes las herramientas necesarias para el desarrollo de un idioma diferente al materno, se orienta hacia la adquisición de técnicas, estrategias, procedimientos, actividades para el desarrollo de competencias lingüísticas en un segundo idioma.

El estudiante adquiere destrezas y habilidades necesarias que le orientan hacia la manera de cómo abordar el segundo idioma con los infantes, para que su desarrollo sea basado en un aprendizaje significativo, es necesario que el futuro docente obtenga los insumos que lo conduzcan hacia aprendizaje interactivo, por ello se hace necesario brindarle las herramientas que se requieran. (MINEDUC, 2013, p. 98).

Las condiciones del uso del término "didáctica" varían según las épocas y las lenguas (Bronckart \& Chiss 2002; Marquilló Larruy, 2001). Del mismo modo, su campo de aplicación cambia en función del contexto lingüístico y del contexto cultural (Dabéne, 1994).

La didáctica de las lenguas se constituyó con el transcurso del tiempo como un discurso crítico sobre la enseñanza, elaborando toda una serie de propuestas e innovaciones. (Dolz Gagnon \& Mosquera, 2009, p.5).
Como prácticas didácticas, Comenio crea situaciones agradables que se adaptan a los intereses de los estudiantes, haciendo uso de su propia lengua, en lugar de una lengua literaria y artificial.

Para identificar la formación didáctica en el abordaje del bilingüismo de los estudiantes utilizamos una entrevista con forma de cuestionario que fue aplicada en la escuela del nivel medio, ciclo diversificado seleccionado para tal fin, durante el desarrollo de las clases de didáctica del segundo idioma L2 (kaqchikel) incluido en el pensum de estudio. El cuestionario incluyó preguntas generadoras de opiniones relativas a la formación didáctica L2 (kaqchikel) en la ENBI Nim Na'oj.

En el cuestionario se les pidió a los estudiantes que explicaran si los docentes aplicaban procesos didácticos bilingües y que si la formación didáctica que recibían era sólida para su desempeño como futuro docente bilingüe. Se analizó las respuestas de los estudiantes puesto que eran preguntas abiertas, se tomó los datos más relevantes de las respuestas que ellos emitieron.

Antes de entregar los cuestionarios a los estudiantes, se grabó solo en audio entrevistas interactivas con el docente que imparte la sub-área de Didáctica de L2 del establecimiento para interiorizar del contexto sociocultural y educacional en el que los estudiantes estaban inmersos.

En relación con ellas, se destaca el apoyo recibido en la obtención de la información sobre la realidad sociolingüística del estudiantado y sobre las metodologías de enseñanza del idioma Kaqchikel en aula bilingüe. Asimismo, el proceso de educación bilingüe se desarrolla de manera incompleta, utilizando solo el idioma español, ya que Mugrabi (2019) dice: "el bilingüismo conlleva un potencial comunicativo que no puede ser evaluado por medio de una sola lengua" (p. 128).

\section{RESULTADOS}

Las ideas y opiniones obtenidas en la entrevista se presentan de la siguiente forma: ¿Desarrolla procesos didácticos bilingües el docente en el abordaje de la L2 (kaqchikel)? En relación a las respuestas de esta primera pregunta: El desarrollo de los procesos didácticos bilingües del docente en el abordaje de la L2 (kaqchikel), de los 27 estudiantes entrevistados el 85\% expresaron que el docente sí desarrolla procesos bilingües y el $15 \%$ indicaron que no.

Entre los procesos didácticos que desarrolla el docente, se auxilia de las siguientes actividades de aprendizaje: canciones, rondas infantiles, hojas de trabajo, elaboración de material didáctico, ejercicios orales, lecturas, dinámicas, cuentos infantiles, poemas, juegos y ejercicios ortográficos. 
De los 27 estudiantes entrevistados respondieron a esta pregunta: Los procesos didácticos que utiliza el docente para el desarrollo de las capacidades en la L2 (kaqchikel) eleva su nivel de bilingüismo; el 52\% manifestaron que los procesos didácticos que se desarrollan en L2, sí elevan su nivel de bilingüismo, el $8 \%$ dijeron que muy poco y el $40 \%$ expresaron que no ayuda a elevar su nivel de bilingüismo.

En relación al uso que el docente le da al Currículum Nacional Base sobre la didáctica de $\mathrm{L} 2$, el $70 \%$ de los estudiantes manifestaron que el docente únicamente lo utiliza para planificar sus clases en el desarrollo de las competencias de área, el $30 \%$ indicaron que el docente de esta sub-área no utiliza el CNB, ni para el desarrollo de los contenidos.

En el caso de los procesos didácticos de L2, donde se perciben el fortalecimiento de la educación bilingüe intercultural; las respuestas se centraron en nombrar actividades, tales como: dramatizaciones, exposiciones, juegos, dinámicas, canciones, cuentos, carteles, en los ejercicios orales y escritos, lecturas, pruebas cortas, trabalenguas en idioma español (L1). Por otra parte, los estudiantes expresaron que no hablan el idioma kaqchikel, razón por la cual no entienden mucho lo que dice el docente en kaqchikel

Referente a los aspectos que contribuyen en la formación didáctica bilingüe para el desarrollo de las habilidades lingüísticas, los estudiantes indicaron que contribuye en la realización de materiales didácticos, para el aprendizaje de escribir palabras en kaqchikel y en la comprensión, aunque hablar en kaqchikel les dificulta. Han aprendido a escribir muy pocas palabras ya que el kaqchikel es muy complejo, en la mayor parte solo les ayuda a leer, pero no comprenden lo que leen y utilizan algunas expresiones orales como los saludos y de agradecimiento.

En cuanto a los contenidos relacionados a la formación didáctica bilingüe en el CNB, los 27 estudiantes entrevistados respondieron que no son suficientes o quizás no son los adecuados, porque no aprenden mucho ya que las competencias deben ser más orientadas a la modalidad bilingüe. Pero la mayoría de los estudiantes son monolingües, por lo que plantean la necesidad de buscar contenidos fundamentales que realmente fortalezca la preparación profesional y que el desarrollo sea más de forma bilingüe.

Las estrategias metodológicas bilingües que utiliza el docente en el rescate y uso del idioma kaqchikel (L2), mencionaron: los juegos, dinámicas de forma oral y escrita, elaboración de materiales didácticos, conversaciones y la convivencia entre compañeros bilingües y monolingües. También mencionaron la importancia de la comunicación entre estudiantes y docente en kaqchikel y las frases que se utilizan en la vida cotidiana se debe hacerlo en kaqchikel.

De los 27 estudiantes entrevistados, el $85 \%$ manifestaron que la formación didáctica, no es sólida para su desempeño como docente bilingüe, ya que se debe aplicar más el bilingüismo que desarrollen la capacidad de los estudiantes en comunicarse en dos idiomas, aunque no lo dominen y los períodos de clases se debe aumentar a 5 períodos, porque 3 no son suficientes. Por consiguiente, no se sienten capaces de dar clases en los dos idiomas puesto que el docente que imparte la Didáctica de L2 no les habla en kaqchikel, solo en algunas ocasiones. En el caso del $15 \%$ de los estudiantes indicaron que la formación didáctica, sí es sólida, pero el detalle está en la capacidad de cada estudiante y depende del empeño que le ponga en el aprendizaje del idioma kaqchikel, se sienten capaces solo en lo básico, pero no más.

En relación al docente, indicó que no era posible desarrollar una educación bilingüe ya que la mayoría de los estudiantes son monolingües españoles y eso dificulta el desarrollo de una educación bilingüe, aunque se realizan intentos, pero por el contexto sociocultural, se dificulta el abordaje del bilingüismo.

\section{CONCLUSIONES}

Las conclusiones de este estudio de investigación no parecenser demasiado alentadoras.

a. En el trabajo de investigación se comprobó que los fundamentos didácticos que proponen los documentos que utiliza el docente en el desarrollo de las capacidades para la enseñanza de la L2 (kaqchikel) carece de la aplicación de estrategias en los procesos de formación didáctica que reciben los estudiantes de magisterio infantil bilingüe intercultural de la Escuela Normal Bilingüe Intercultural Nim Na'oj, debido a que los documentos están elaborados desde un punto de vista monolingüe (español) y también la formación monolingüe deldocente en servicio, por lo que las actividades que realizó en el aula, lo hizo desde un solo enfoque, una sola visión, por ello prevalecieron procesos habituales: leer, cantar y repetir las frases o palabras.

b. El estudio muestra que los contenidos fundamentales en la sub-área de Didáctica del Segundo Idioma L2 (kaqchikel) el docente utiliza el CNB como una herramienta para orientar los procesos de enseñanzaaprendizaje, pero no para el desarrollo de los contenidos de didáctica de $\mathrm{L} 2$, en este sentido el CNB no ha sido un medio eficaz, debido a que el CNB se enfoca en competencias monolingües y no tiene una 
visión bilingüe, así también porque el docente no tienen el dominio de la segunda lengua kaqchikel. Por lo tanto, desarrolla procesos de enseñanza- aprendizaje "semibilingües", con un $75 \%$ en idioma español. Consecuentemente, los estudiantes no desarrollan las habilidades comunicativas en la segunda lengua L2 (kaqchikel), y tienen pocas prácticas orales en los cantos, juegos, trabalenguas y pequeñas lecturas.

c. El docente no cuenta con una metodología definiday técnicas para el desarrollo de los contenidos didácticos propuestos en la sub-área de didáctica en L2 y de la integración del resto de destrezas de lecturay escritura para el fortalecimiento de la formación delos estudiantes de Magisterio Infantil Bilingüe Intercultural.El docente hace el intento de aplicar procesos metodológicos bilingües para orientar a los futuros docentes, pero no ha sido posible porque el tiempo que tardan los períodos de clases no son suficientes para aplicar las diferentes técnicas y una metodologíainnovadora y adecuada para una formación eficaz.

d. Los docentes tienen poco conocimiento de cómo implementar procesos didácticos bilingües en la ENBI, las pocas herramientas didácticas que aplicaron no fueron suficientes y eficientes para el fortalecimiento bilingüe.

\section{REFERENCIAS BIBLIOGRÁFICAS}

Andrade R. (2008). Didáctica Sistémica, ISSN 1809-3108, volumen 8. Universidad Federal Do Río Grande-FURG. Revista.

Bronckart, J.-P. \& Chiss, J.-L. (2002). artículos "Didáctique (a)", "Didactique des disciplines (b)", “Didactique de la lengue maternelle (c)”. Encyclopedia Universal.
Bobenrieth A. M.A. (1998). "Escritura y lectura crítica de artículos científicos", En: Burgos Rodriguez R. Metodología de investigación y escritura científica en clínica. Parte IV. Granada. Escuela Salud Pública.

Dolz, Garnon \& Mosquera: (2009). La didáctica de las lenguas: una disciplina en proceso de construcción. Universidad de Ginebra (Suiza).

Henriquez E. \& M. I. Zepeda: (2004). Elaboración de un Artículo Científico de Investigación. Departamento Enfermería. Universidad de Concepción, Concepción, Chile.

\section{Sobre autora}

\section{Lilian Esterlina Chalí Sareh}

Es Profesora de Inglés y otras áreas y sub-áreas en la ENBI NIM NA'OJ, de San Martín Jilotepeque (desde hace 10 años), licenciada en la Enseñanza del Idioma Español y la Literatura, egresada de la EFPEM-Universidad de San Carlos de Guatemala, Maestría en Educación Bilingüe Intercultural en Pedagogía de Texto por la Universidad de San Carlos de Guatemala y Niños del Mundo, línea de investigación Principal: El Bilingüismo (KaqchikelEspañol).

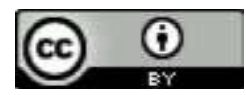

Este texto está protegido por una licencia Creative Commons 4.0.

Usted es libre para Compartir - copiar y redistribuir el material en cualquier medio o formato - y Adaptar eldocumento - remezclar, transformar y crear a partir del material- para cualquier propósito, incluso comercialmente, siempre que cumpla la condición de:

Atribución: Usted debe reconocer el crédito de una obra de manera adecuada, proporcionar un enlace a la licencia,e indicar si se han realizado cambios. Puede hacerlo en cualquier forma razonable, pero no de forma tal que sugieraque tiene el apoyo del licenciante o lo recibe por el uso que hace. 